\title{
ON THE DEVELOPMENT OF ICME TOOLS FOR CREEP AND AGING OF CMSX®-8
}

\author{
E.A. Estrada Rodas ${ }^{1}$, S. Gorgannejad ${ }^{1}$, R.W. Neu ${ }^{1,2}$, Z. Dyer ${ }^{3}$, P.M. Draa ${ }^{4}$, and S.R. Shinde ${ }^{3}$ \\ ${ }^{1}$ The George W. Woodruff School of Mechanical Engineering, Georgia Institute of Technology, Atlanta GA \\ ${ }^{2}$ School of Materials Science and Engineering, Georgia Institute of Technology, Atlanta, GA \\ ${ }^{3}$ Siemens Energy Inc., Orlando FL \\ ${ }^{4}$ Siemens Energy Inc., Charlotte, NC
}

Keywords: aging, creep, crystal viscoplasticity, diffusivity, Ni-base superalloy, rafting

\begin{abstract}
In this paper three different tools are developed to aid Integrated Computational Materials Engineering (ICME) of Ni-base superalloys for next generation industrial gas turbines: (i) models to predict the state of the $\gamma / \gamma^{\prime}$ structure through modeling of the kinetics of aging, including both rafting and coarsening, (ii) a crystal viscoplasticity (CVP) model for determining the creep and cyclic deformation response that uses the current state of the $\gamma / \gamma^{\prime}$ structure as is an input, and (iii) the use of computational thermodynamics and kinetics software for multi-component systems to determine how diffusivity, which is a key parameter in the aging and in viscoplasticity models, is altered with variations in composition of the $\gamma$ and $\gamma^{\prime}$ phases. Experiments are conducted on a reduced Re content superalloy, CMSX®-8, to determine the parameters of the aging and crystal viscoplasticity models.
\end{abstract}

\section{Introduction}

During this past decade, next generation industrial gas turbines (IGT) have been moving toward the employment of single crystal blades, which are now ubiquitous in aerospace gas turbine propulsion systems. Second generation single crystal (SX) superalloys commonly used in current generation aerospace propulsion systems (CMSX-4®, PWA1484, René N5) contain 3 wt $\%$ Re, which reduces creep deformation in the $\gamma$ channels and maintains the stability of the microstructure. Consequently, these second generation superalloys have become the benchmark in the development of superalloys more suitable for IGT applications that are characterized by considerably larger blade sizes and cross sections and longer operational times. The cost of the rare elements, specifically $\mathrm{Re}$, is one of the limiting constraints in selecting these superalloys for IGT applications. In addition, there are challenges in developing casting protocols for these larger sectioned components that have propensity for developing casting anomalies and microstructure variations through the blade and its platform. Consequently, there is a large push to develop SX superalloys with no or reduced Re content that have properties suitable for IGT applications.

Recently Cannon-Muskegon introduced a SX superalloy, denoted CMSX®-8, with Re reduced to 1.5 wt.\%, but otherwise similar in composition to the benchmark superalloy CMSX-4® [1]. Initial mechanical property studies conducted on this new superalloy show minimal reduction in creep and fatigue properties compared to the benchmark [1]. Since IGT must operate at sustained loads and temperatures for extremely long times, the aging of the alloy and its impact on mechanical behavior including long-term creepfatigue interactions and thermomechanical fatigue needs to be well understood and predicted when designing components. It is thought that the Re plays a role in this long-term behavior and initial studies to date cannot readily capture these effects.
This paper explores the development of integrated computational materials engineering (ICME) tools for predicting the impact of reducing $\mathrm{Re}$ on the degradation and mechanical behavior of these superalloys under service conditions. In particular, this study is aimed at expanding knowledge of longer-term degradation of CMSX®-8 through aging and characterization studies. The end goal is to develop a microstructure-sensitive constitutive model that can be used to predict the response of components under realistic IGT conditions. This project is split into several facets. The first one involves establishing the kinetics of aging of the microstructure with the objective of determining how rapidly the microstructure evolves under service conditions and developing models that can predict the state of the $\gamma / \gamma^{\prime}$ structure. The second facet consists of developing a physics-based constitutive model that captures relevant microstructure attributes to enable thermomechanical analysis of realistic geometries. The third facet consists of exploring the use of thermodynamics and kinetics software such as Thermo-Calc and DICTRA (DIffusion Controlled TRAnsforamtions), to model diffusion-controlled microstructure evolution and to determine how variations in the elemental composition, both in $\gamma$ channels and $\gamma^{\prime}$ particles, affect the diffusivity that is implicitly embedded in the coefficients of the rate equation in the crystal viscoplasticity model. The aim is to explore whether the variances in the long-term creep and aging behavior due to reducing the $\mathrm{Re}$ content can be captured in constitutive models by accounting for differences in diffusivity in the aging kinetics and flow rule.

\section{Material description}

The material investigated is $\mathrm{CMSX}-8[\mathrm{~B} / \mathrm{C}]$ which contains intentional additions of boron and carbon to improve castability and machinability [2]. The nominal composition of this alloy can be found in reference [3]. The CMSX-8 rods used in this research were cast by PCC Airfoils and then solution and double age treated using proprietary conditions typical for blade components. The initial $\gamma / \gamma^{\prime}$ microstructure of this alloy is shown in Figure 1.

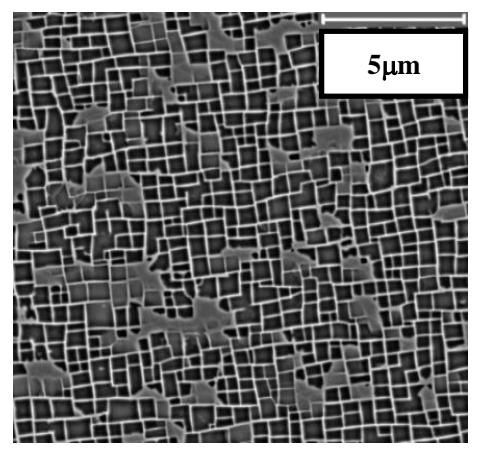

Figure 1. SEM image of as-received microstructure of CMSX-8. 
In this work, for simplicity we will refer to $\mathrm{CMSX}-8[\mathrm{~B} / \mathrm{C}]$ as CMSX-8. The volume fractions of the $\gamma$ and $\gamma^{\prime}$ phases were estimated to be 0.27 and 0.73 , moreover the average $\gamma$ channel width and $\gamma^{\prime}$ size, were estimated to $0.14 \mu \mathrm{m}$ and $0.74 \mu \mathrm{m}$, respectively, these values were obtained using techniques derived from two-point statistics $[4,5]$. The dendritic arm spacing and the secondary dendritic arm spacing were measured using SEM images, and were estimated to $436 \pm 150 \mu \mathrm{m}$ and $112 \pm 37 \mu \mathrm{m}$, respectively which are comparable to the measured dendritic arm spacing of other Ni-based superalloys $[6,7]$.

\section{Aging studies on the <001> oriented single crystal CMSX-8}

During high temperature service conditions (the temperature of the gas stream is about $1477{ }^{\circ} \mathrm{C}$ [8]), single crystal gas turbine blade materials, subjected largely to centrifugal forces resulting from significant rotational speeds, are prone to severe microstructural degradation. The combined influence of stress and temperature, results in an evolution of the $\gamma / \gamma^{\prime}$ microstructure to a rafted microstructure marked by horizontal or vertical $\gamma$ channels widening and $\gamma^{\prime}$ precipitates elongation and coalescence which ultimately leads to topological inversion of the microstructure. The mechanisms of failure under creep in an evolved microstructure will differ from the initial microstructure and therefore understanding the kinetics of this process is important. In this work, the kinetics of microstructural evolution of CMSX-8 under high temperature and low stress levels that result in a rafted microstructure is characterized by an empirical model adopted from Epishin et al. for CMSX-4® [9].

The thermodynamic driving force for rafting is impacted by the applied stress $(\sigma)$ and by the misfit stresses as well as the anisotropy of the resulting dislocation debris fields. The misfit stresses are induced by the $\gamma$ and $\gamma^{\prime}$ lattice parameter difference, denoted as lattice misfit, and is defined by [10],

$$
\delta=\frac{2\left(a_{\gamma^{\prime}}-a_{\gamma}\right)}{a_{\gamma^{\prime}}+a_{\gamma}}
$$

where $a_{\gamma}$ and $a_{\gamma}$ are $\gamma^{\prime}$ precipitate and $\gamma$ channel lattice parameters, respectively. Cubic precipitates in alloys with negative lattice misfit elongate perpendicular to the loading direction under tensile stress (N-type rafts) while they coalesce in loading direction under compressive stresses (P-type rafts). Most modern Ni-base superalloys, including CMSX-8, used in hot gas path sections are negative misfit alloys [10].

Experimental procedure and microstructure characterization To investigate the kinetics of microstructure degradation as a function of applied stress and temperature, specimens with a tapered geometry and circular cross section were used to create a linear stress gradient along the sample. The cross section diameter varies from $12.7 \mathrm{~mm}$ to $6.35 \mathrm{~mm}$ within a $50.8 \mathrm{~mm}$ length. Hence the applied stress level changes by a factor of four at the two ends of the specimen. This method provides the means to rapidly characterize the stress dependence of microstructure evolution in a single specimen. To examine the $\gamma / \gamma^{\prime}$ microstructure within the core dendritic region, microscopy was performed using a TESCAN MIRA3 XM SEM at $10 \mathrm{kV}$. For image quantification purposes, the two-point correlation technique was used. The twopoint correlation functions are statistical microstructure descriptors that provide the tools to characterize spatial arrangement and anisotropy of microstructural attributes. The two-point correlation function captures the probability density that a randomly located vector of length $\boldsymbol{r}$ has its head and tail in a specific local sate and is mathematically represented by [11],

$$
f\left(h, h^{\prime} \mid \mathbf{r}\right)=\frac{\iiint_{x \in \Omega \mid \mathbf{r}} m(\mathbf{x}, h) m\left(\mathbf{x}+\mathbf{r}, h^{\prime}\right) d \mathbf{x}}{\operatorname{vol}(\Omega \mid \mathbf{r})}
$$

where $\Omega$ is the spatial domain, $h$ is the local state of interest and $m$ is the microstructure function representing the volume density of local state that is normalized by the volume of the region. The microstructural features of interest in this study are size and morphology of $\gamma$ channels and $\gamma^{\prime}$ precipitates. A MATLAB ${ }^{\circledR}$ routine was developed that uses digital binary images and processes them by two-point correlation method. Quantification of microstructures was performed on specimens subjected to isothermal aging under different stress levels and dwell times. Epishin et al. [9] proposed that $\gamma$ channel widening during rafting represents the degree of microstructure degradation under stressassisted aging condition. Therefore, measurement of kinetics of channel widening provides the means to characterize the extent microstructure evolution by rafting.
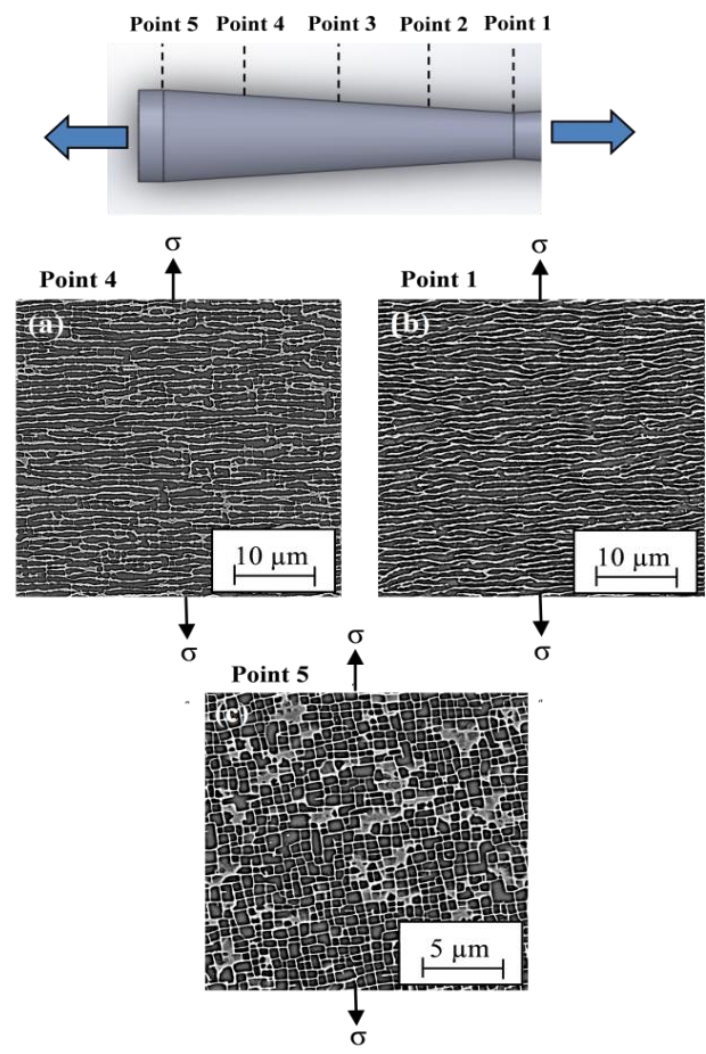

Figure 2. SEM images showing different stages of rafting:

(a) $34 \mathrm{MPa}, 1120^{\circ} \mathrm{C}, 50$ hours, (b) $100 \mathrm{MPa}, 1120^{\circ} \mathrm{C}, 50$ hours, (c) $38 \mathrm{MPa}, 950^{\circ} \mathrm{C}, 445$ hours.

Mechanism of rafting

By application of an external tensile stress along the $\gamma^{\prime}$ cube axis, the primary slip systems in the $\gamma$ channels are activated and $a / 2<110>\{111\}$ creep dislocations begin to glide in the channels. The movement of the dislocations is under the influence of the applied stress and the misfit stresses simultaneously. In absence of external stress, misfit stresses at the $\gamma / \gamma^{\prime}$ horizontal and vertical 
interfaces are equally distributed. However, the application of external stress will induce a gradient of misfit stresses between the faces of the same precipitate in the vertical and in the highly stressed horizontal channels. The influence of mobile dislocations in the $\gamma$ channels generally relieves these misfit stresses. Dislocations glide from locations with low misfit stress to locations with high misfit stress and are then absorbed by the horizontal $\gamma / \gamma^{\prime}$ interfaces. The formation of dislocation networks at the interface results in loss of coherency which causes relaxation of the misfit stresses in the horizontal channels while the vertical channels remain coherent $[12,13]$. Rafting is a sequential process and requires a certain amount of plastic deformation $\left(0.1 \%\right.$ for CMSX-4® at $950{ }^{\circ} \mathrm{C}$ [14]) in the $\gamma$ channel, glide of creep dislocations and subsequent loss of coherency. Dislocation networks provide the diffusional path for atoms to travel. The driving force for mass transport is driven by the chemical potential gradient causing the interchange of the neighboring atoms. Therefore, $\gamma$ partitioning alloying elements such as $\mathrm{Cr}, \mathrm{Co}$, and $\mathrm{Re}$ diffuse to the horizontal channels and on the other hand $\gamma^{\prime}$ partitioning elements such as $\mathrm{Al}$ and $\mathrm{Ti}$ travel to vertical channels [15].

Isothermally aged microstructures are shown in Figure 2. Because of the negative lattice misfit under tensile stress, elongation of the precipitates has occurred normal to the loading direction and the rafted microstructure is clearly observed, particularly when the temperature is sufficiently high as in Figure 2(a) and 2 (b). At the lower temperature of $950{ }^{\circ} \mathrm{C}$ with dwell time of 445 hours and similar stress level as in Figure 2(a), there is no apparent morphology change (Figure 2(c)). Rafting is a diffusioncontrolled process which is thermally activated. Consequently, the impact of temperature on the state of microstructure is more pronounced.

The volume fraction of the $\gamma^{\prime}$ precipitates remains constant as the morphology changes. This has been addressed by a number of authors experimentally and computationally [9, 12, 13]. The elongation of the precipitates in the horizontal direction is associated with its shrinkage in the vertical direction and therefore widening of the $\gamma$ channels is imposed geometrically. The shrinkage of the vertical channels, which is compensated by the horizontal channel widening, proceeds until the vertical channels disappear completely and the microstructure reaches the fully rafted state (Figure 2 (b)). Extensive rafting and formation of links between precipitates will eventually lead to the inversion of the $\gamma / \gamma^{\prime}$ microstructure such that the discontinued disordered $\gamma$ phase is distributed in the ordered $\gamma^{\prime}$ matrix phase which substantially affects the activity of the dislocations [10].

\section{Kinetics of rafting}

The experimental results from rafting tests have been shown to follow a phenomenological Arrhenius type relation [9],

$$
\dot{w}_{\text {raft }}(T, \sigma)=A \exp \left(-\frac{Q-U(T) \sigma}{k T}\right)
$$

where $\mathrm{A}$ is a pre-exponential factor, $\mathrm{Q}$ is the rafting activation energy, $\sigma$ is applied stress, $k$ is universal gas constant, $\mathrm{T}$ is the absolute temperature and $\mathrm{U}(\mathrm{T})$ is temperature dependent function defined as,

$$
U(T)=U_{T}\left\langle T-T_{o}\right\rangle^{n}
$$

The exponent $n$ and the coefficient $U_{T}$ are fit to experiments. The reference temperature $T_{o}$ is the temperature below which no rafting occurs. For a best fit, this value for CMSX-8 was found to be $850{ }^{\circ} \mathrm{C}$ which is close to the CMSX-4® reference temperature of $828^{\circ} \mathrm{C}$ [9].

Figure 3 shows the increase in channel widening rate versus stress at temperatures of $900^{\circ} \mathrm{C}, 950^{\circ} \mathrm{C}$, and $1120^{\circ} \mathrm{C}$. The model parameters are given in Table I. A rafting activation energy of $206 \mathrm{~kJ} / \mathrm{mol}$ is calculated for CMSX-8. This value is lower than the rafting activation energy for CMSX-4® (222 kJ/mol) [9] and is consistent with the greater Re content of CMSX-4®.

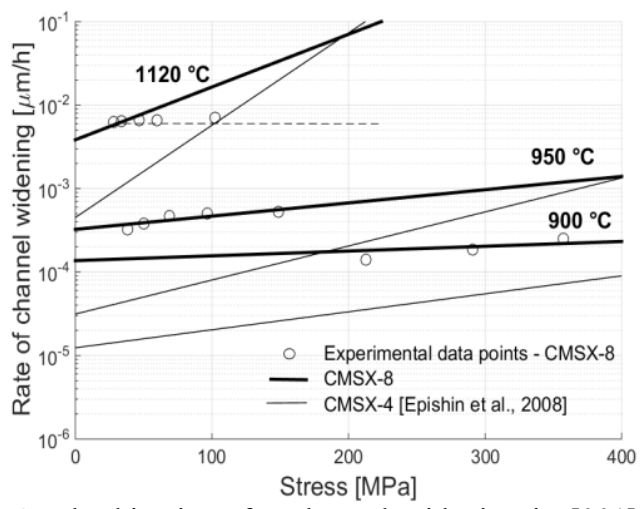

Figure 3. The kinetics of $\gamma$ channel widening in [001] oriented single crystal, CMSX-8.

Table I. Rafting kinetics model parameters, CMSX-8

\begin{tabular}{|c|c|c|c|}
\hline $\mathrm{A}\left(\mu_{\mathrm{m}} / \mathrm{h}\right)$ & $\mathrm{Q}(\mathrm{kJ} / \mathrm{mol})$ & $\mathrm{U}_{\mathrm{T}}\left(\mathrm{J} / \mathrm{mol} \cdot \mathrm{MPa} \cdot \mathrm{K}^{\mathrm{n}}\right)$ & $\mathrm{n}$ \\
\hline $2.0 \times 10^{5}$ & 206 & 0.033 & 1.525 \\
\hline
\end{tabular}

The model is in good agreement with the experimental data except at the highest temperature of $1120{ }^{\circ} \mathrm{C}$. At this temperature, the experimental data points follow the horizontal dashed line which represents a saturation level of the microstructure evolution as the fully rafted microstructure is reached at all stress levels. Beyond saturation level, rafting is decelerated and isotropic coarsening becomes the dominant process. It is suggested that the approximate value of $3 w_{0}$ for channel width size is the criterion which defines the completion of rafting [9]. This gives the final channel width equal to $0.42 \mu \mathrm{m}$ for CMSX-8.

\section{Crystal viscoplasticity model for creep}

In this section a physics-based crystal viscoplasticity (CVP) model is described that is capable of predicting the creep deformation response of CMSX-8. To capture the highly anisotropic inelastic behavior of single crystal alloys, at each material point, the orientation of the crystal lattice is prescribed to track the collective dislocation motion on each slip system, explicitly defined by a slip plane and a slip direction [16]. In general for Ni-base superalloys, three families of slip systems may be activated in the $\gamma$ and $\gamma^{\prime}$ phases in face-centered cubic lattices, these include the 12 primary octahedral slip systems $\langle 110\rangle\{111\}$, the 12 secondary octahedral slip systems $\langle 112>\{111\}$ (shear acting on partial dislocations in $\gamma^{\prime}$ precipitates), and 6 cube slip systems $\langle 110\rangle\{001\}$. The primary octahedral slip occurs on the close-packed planes and is active over the entire temperature range. The secondary octahedral slip systems are important to consider when conditions are ripe for dislocations to cut through the $\gamma^{\prime}$ precipitates since dislocations split into Shockley partial dislocations and the resolved shear stress on the partials becomes an important driver. Macroscopic cube slip may only be active at 
higher temperatures. Microscopic cube slip has been observed only occasionally while zig-zag octahedral slip in the $\gamma$ channels is a major source of macroscopic cube slip [17]. Since only the $<001>$ crystallographic orientation is examined in the current investigation, only dislocation motion to the primary and secondary slip systems will be addressed.

\section{Constitutive relations and flow rules}

In continuum crystal plasticity formulations -where symbols in bold represent tensors- the inelastic part of the deformation gradient $\boldsymbol{F}^{\text {in }}$ is determined from the macroscopic inelastic velocity gradient which is the aggregate sum of the inelastic shear strain rate $\dot{\gamma}^{i n(\alpha)}$ on all possible slip systems $\alpha$,

$$
\mathbf{L}^{i n}=\dot{\mathbf{F}}^{i n} \mathbf{F}^{i n^{-1}}=\sum_{\alpha=1}^{N_{\text {slip }}} \dot{\gamma}^{i n(\alpha)}\left(\hat{\mathbf{d}}_{o}^{(\alpha)} \otimes \hat{\mathbf{n}}_{o}^{(\alpha)}\right)
$$

where $\hat{\mathbf{d}}_{o}^{(\alpha)}$ and $\hat{\mathbf{n}}_{o}^{(\alpha)}$ are the slip direction and slip plane normal unit vectors, respectively, defined in the reference configuration for each slip system, and $N_{\text {slip }}$ is the number of possible active slip systems. In Ni-base superalloys single crystals, dislocation slip is either limited to the $\gamma$ channels or cutting through the $\gamma^{\prime}$ particles. Consequently, the driver for inelastic shear strain rate is different whether the $\gamma^{\prime}$ precipitate can be cut or not. Therefore the inelastic velocity gradient is additively decomposed into two terms corresponding to the relevant deformation mechanisms that are active at each phase:

$$
\mathbf{L}^{i n}=f_{\gamma}\left(\sum_{\alpha=1}^{12} \dot{\gamma}_{\gamma}^{i n(\alpha)}\left(\hat{\mathbf{d}}^{(\alpha)} \otimes \hat{\mathbf{n}}^{(\alpha)}\right)\right)+f_{\gamma^{\prime}}\left(\sum_{\alpha=13}^{24} \dot{\gamma}_{L 1_{2}}^{i n(\alpha)}\left(\hat{\mathbf{d}}^{(\alpha)} \otimes \hat{\mathbf{n}}^{(\alpha)}\right)\right)
$$

where $f_{\gamma}$ and $f_{\gamma}$ are the volume fractions of the $\gamma$ and $\gamma^{\prime}$ phases, respectively. Here, the slip plane is assumed to be octahedral for all slip systems; i.e., $\hat{\mathbf{n}}^{(\alpha)} \|\{111\}$. Therefore in $\gamma$, the primary octahedral slip systems operate, $\hat{\mathbf{d}}^{(\alpha)} \|\langle 110\rangle$, while dislocations cutting through $\gamma^{\prime}$ depend on shear stress acting on partial dislocations necessary to cut through the ordered structure that manifests as ribbon shearing; hence, $\hat{\mathbf{d}}^{(\alpha)} \|<112>$ for $13 \leq \alpha \leq$ 24. Splitting the dislocation mechanisms in $\boldsymbol{L}^{\text {in }}$ enables the model to be sensitive to volume fraction size and morphology of the $\gamma / \gamma^{\prime}$ microstructure. This is critical for the prediction of the different aspects of the creep response that vary depending on temperature and stress [8, 17-19]. In particular, features of incubation, primary creep, tertiary creep, and rafting can be addressed. Secondary creep appears at the saturation point when the $\gamma$ channels have been completely filled with dislocations [20]. We compared CMSX-8 creep responses to CMSX-4® [8], Figure 4, and found that dominant mechanisms in CMSX-8 also appear to partition into the same temperature and stress regimes as CMSX$4 \circledast$.

Inelastic shear strain rate relations

The physics-based constitutive model is founded on a crystal viscoplasticity and damage formulation originally developed by Ma, Dye, and Reed [19]. In this work, the model is extended to CMSX-8, the backstress is reformulated, and a diffusivity parameter is added to study the effect of composition on creep behavior. Specifically, the effect Re content of the alloy by using an Arrhenius type diffusivity parameter is studied.

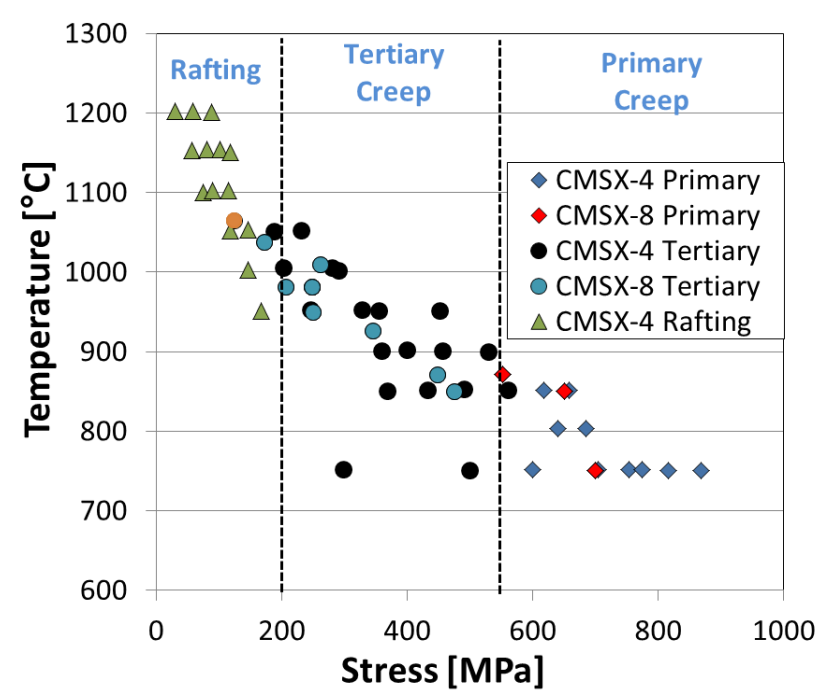

Figure 4. Temperature and stress dependence on the characteristic deformation mechanism.

The relationship between the inelastic strain rates and the density of mobile dislocation is assumed to follow the Orowan equation. In the $\gamma$ channels the flow rule for each primary slip system is described by:

$$
\begin{aligned}
\dot{\gamma}_{\gamma}^{i n(\alpha)}= & \rho_{\gamma}^{(\alpha)} b \lambda_{\gamma}^{(\alpha)} F_{\text {attack }} \Theta(T, \xi) \\
& \exp \left\{\frac{-Q_{\text {slip }}^{110}+\left(\left|\tau_{\text {eff }}^{(\alpha)}\right|-\kappa_{\gamma}^{(\alpha)}\right) V_{c 1}^{(\alpha)}}{k T}\right\} \operatorname{sign}\left(\tau_{\text {eff }}^{(\alpha)}\right)
\end{aligned}
$$

The activation energy $Q_{\text {slip }}^{110}$ represents the thermal component for overcoming the obstacles in absence of stress which is similar to activation energy for self-diffusion. The coefficients before the exponential represents the mobile dislocation density on that slip system, the Burger's vector, and the dislocation vibration frequency, which is less than the Debye frequency, and

$$
\lambda_{\gamma}^{(\alpha)}=c_{j u m p 1}\left(\frac{1}{L_{\gamma}}+\frac{1}{L_{\rho}^{(\alpha)}}\right)^{-1}
$$

defines the dislocation mean free path (jump distance) which is inversely proportional to the dislocation pinning spacing in the $\gamma$ phase, here with components due to pinning at the $\gamma / \gamma^{\prime}$ interfaces (hence, associated to width of $\gamma$ channel) and average spacing of forest dislocations,

$$
L_{\rho}^{(\alpha)}=\frac{c_{F d i s l}}{\sqrt{\rho_{F}^{(\alpha)}}}
$$

The dislocation mean free path is an average measure of the distance travelled by a dislocation between the source and storage points. Based on experimental observations presented by other authors [21-24], a lower-bound limit of 12 times the burgers 
vector has been imposed on $\lambda_{\gamma}^{(\alpha)}$, this accounts for the repulsive force between dislocations and prevents them from becoming infinitely close which would create excessive resistance to deformation. Forest and parallel dislocations are defined as projections of dislocation densities described in ref. [25].

The activation volume is given by

$$
V_{c 1}^{(\alpha)}=c_{v c 1} b^{2} \lambda_{\gamma}^{(\alpha)}
$$

In order to account for the diffusivity of Re during creep, a dimensionless diffusivity parameter which is a function of temperature and composition has been added to the $\gamma$ phase flow rule,

$$
\Theta(T, \xi)=A(T, \xi) \exp \left(-\frac{Q_{o}(\xi)}{k T}\right)
$$

where $\xi$ denotes the parameter is dependent on composition. The pre-exponential coefficient and the activation energy for diffusion are calculated using Thermo-Calc and DICTRA and experimental creep data. This will be addressed in detail in a later section.

The effective resolved shear stress is calculated as the added contributions of the applied loading, the misfit stresses and the backstress due to the bowing of dislocations:

$$
\tau_{e f f_{\gamma}}^{(\alpha)}=\tau^{(\alpha)}+\tau_{m i s}^{(\alpha)}-\chi^{(\alpha)}
$$

The misfit stresses are assumed to relax due to the $\gamma$ dislocations becoming captured in the $\gamma / \gamma^{\prime}$ interface which causes the loss elastic coherency $[19,20]$,

$$
\tau_{m i s}^{(\alpha)}\left(T, \gamma_{\gamma}^{(\alpha)}\right)=\tau_{m i s 0}^{(\alpha)}\left\{1-\exp \left(\left(\frac{\sum_{\alpha=1}^{12} \rho_{\gamma}^{(\alpha)}}{-\rho_{\gamma}^{r e f}}\right)\right)\right\}
$$

The backstress is due to the Orowan stress and relates to the bowing of the dislocations in the $\gamma$ channels between the precipitate interfaces and the forest dislocations and is defined in a similar way as Busso and McClintock [26],

$$
\chi^{(\alpha)}=\left(\frac{c_{\text {oro }} G b}{\lambda_{\gamma}^{(\alpha)}}-\tau_{\gamma}^{(\alpha)}{ }_{\text {pass }}\right) \operatorname{sign}\left(\tau_{\text {eff }}^{(\alpha)}\right)
$$

The threshold stresses arise from the hardening generated by the passing of dislocations,

$$
\kappa_{\gamma}^{(\alpha)}=\tau_{\gamma \text { pass }}^{(\alpha)}
$$

The passing stress is dependent on the parallel dislocation density within the $\gamma$ phase (Taylor self-hardening),

$$
\tau_{\gamma}^{(\alpha)}{ }_{\text {pass }}=c_{\text {pass } 11} G b \sqrt{\rho_{P}^{(\alpha)}}
$$

In the $\gamma^{\prime}$, the flow rule defines the rate of dislocation movement through the $\gamma^{\prime}$ precipitates in a ribbon shear fashion on the secondary slip systems,

$$
\begin{aligned}
\dot{\gamma}_{L 1_{2}}^{\text {in }(\alpha)}= & \rho_{L 1_{2}}^{(\alpha)} b \lambda_{L 1_{2}}^{(\alpha)} F_{\text {attack }} \\
& \exp \left\{\frac{-Q_{\text {slip }}^{112}+\left(\left|\tau^{(\alpha)}\right|-\kappa_{L 1_{2}}^{(\alpha)}\right) V_{c 2}^{(\alpha)}}{k T}\right\} \operatorname{sign}\left(\tau^{(\alpha)}\right)
\end{aligned}
$$

the dislocation mean free path (jump distance) is controlled by the size of the $\gamma^{\prime}$ particles $L_{\gamma^{\prime}}$ and the interaction with forest dislocations in the $\gamma$ phase,

$$
\lambda_{L 1_{2}}^{(\alpha)}=c_{j u m p 2}\left(\frac{1}{L_{\gamma^{\prime}}}+\frac{1}{L_{\rho}^{(\alpha)}}\right)^{-1}
$$

a lower-bound limit is also imposed on the dislocation spacing of the $\gamma^{\prime}$ phase. A close form solution of two strongly-coupled dislocations entering a precipitate is used for this limit [8],

$$
\lambda_{\min }=\frac{G b^{2}}{\pi \gamma_{A P B}}
$$

The threshold stresses are a result of the hardening generated by the passing of dislocations and the resistance created from the anti-phase boundary energy,

$$
\kappa_{L 1_{2}}^{(\alpha)}=\tau_{L 1_{2} \text { pass }}^{(\alpha)}+\tau_{A P B}
$$

The passing stress is defined in a similar way as before,

$$
\tau_{L 1_{2} \text { pass }}^{(\alpha)}=G b c_{p a s s 22} \sqrt{\rho_{P}^{(\alpha)}}
$$

Finally the anti-phase boundary resistance is described by the anti-phase boundary energy:

$$
\tau_{A P B}=\frac{\gamma_{A P B}}{b}
$$

Evolution equations and damage parameter

Internal state variables (ISVs) that evolve with deformation and are tracked on each slip system can be either phenomenologicalbased [18, 27-31] or dislocation-based [26, 32-34]. Dislocationbased formulations better address the physical process of deformation, though often adding some level of complexity. Additionally, evolution equations may couple mass diffusion/microstructure evolution kinetics to capture microstructural evolution [35-39]. For dislocation-based CVP models, the dislocation density is the primary ISV. The dislocation density in the $\gamma$ channels, in the $\gamma^{\prime}$ particles and in the 
$\gamma / \gamma^{\prime}$ interface are controlled by different set of evolution equations since their values are often distinctly different, particularly under conditions when looping of the precipitates occurs, and hence the microstructure attributes that control their evolutions are distinct. In the $\gamma$ channels, the dislocation density based strain hardening uses the Kocks-Mecking form [40],

$$
\dot{\rho}_{\gamma}^{(\alpha)}=\frac{c_{m u l t 1}}{b \lambda_{\gamma}^{(\alpha)}}\left|\dot{\gamma}_{\gamma}^{i n(\alpha)}\right|-c_{a n n h 1} \rho_{\gamma}^{(\alpha)}\left|\dot{\gamma}_{\gamma}^{i n(\alpha)}\right|
$$

This equation assumes evolution depends solely on selfhardening; i.e., hardening depends on the dislocation density on each individual slip system only. The first term represents the multiplication mechanism which is accommodated by additional mobile dislocations with fixed travel distance and the second term represents dynamic recovery associated with the annihilation of parallel dislocations with anti-parallel Burgers vectors within a critical distance.

In the $\gamma^{\prime}$ particles, the evolution equation includes one more multiplication term that captures the ease of a dislocations entering the $\gamma^{\prime}$ particle, controlled by a thermally activated process

$$
\dot{\rho}_{L 1_{2}}^{(\alpha)}=c_{\text {mult } 21} \rho_{p b}^{(\alpha)} \Gamma+\frac{c_{m u l t 22}}{b \lambda_{\gamma^{\prime}}^{(\alpha)}}\left|\dot{\gamma}_{\gamma^{\prime}}^{(\alpha)}\right|-c_{\text {annh } 2} \rho_{\gamma^{\prime}}^{(\alpha)}\left|\dot{\gamma}_{\gamma^{\prime}}^{(\alpha)}\right|
$$

where,

$$
\left\{\begin{array}{l}
\Gamma=F_{\text {attack }} \exp \left\{\frac{-Q_{\mathrm{climb}}^{p b}+\left(\left|\tau^{(\alpha)}\right|-\tau_{p b_{\text {pass }}}^{(\alpha)}\right) V_{c 2}^{(\alpha)}}{k T}\right\} \\
Q_{\mathrm{climb}}^{p b}=c_{\text {misfit }} G b^{3}|\delta| \\
\tau_{p b_{\text {pass }}}^{(\alpha)}=c_{\text {pass } 21} G b \sqrt{\rho_{P_{p b}^{(\alpha)}}^{(\alpha)}}
\end{array}\right.
$$

The first term of equation (24) considers the contribution from the dislocations that are generated in the channels and then gather at the phase boundary ( $\gamma / \gamma^{\prime}$ interface). Enough dislocations need to gather at the interface to start cutting the precipitates. The formation of these dislocations increases the incoherency of the precipitates making them harder to be cut, this is accounted for using by the interface passing stress $\tau_{p b_{\text {pass }}}^{(\alpha)}$ and is a function of parallel dislocation density at the $\gamma / \gamma^{\prime}$ interface.

The evolution equations for dislocations gathered at the interface also uses the Kocks-Mecking form and is dependent on the shearing rates in the $\gamma$ channels:

$$
\dot{\rho}_{p b}^{(\alpha)}=\frac{c_{m u l t}^{p b}}{b L_{\gamma}}\left|\dot{\gamma}_{\gamma}^{i n(\alpha)}\right|-c_{a n n h}^{p b} \rho_{p b}^{(\alpha)}\left|\dot{\gamma}_{\gamma}^{i n(\alpha)}\right|
$$

Damage is accounted for through the void condensation generated by the annihilation of dislocations in the $\gamma$ phase, the $\gamma^{\prime}$ phase and in the $\gamma / \gamma^{\prime}$ interface. It is assumed that a single vacancy is $b^{3}$ in volume, and that the evolution equations for the radius of the voids are proportional to the annihilation terms in the evolution of the dislocation densities:

$$
\left\{\begin{array}{l}
\dot{R}_{\gamma}^{(\alpha)}=c_{\text {void } 11} \frac{c_{a n n h 1} \rho_{\gamma}^{(\alpha)}\left|\dot{\gamma}_{\gamma}^{i n(\alpha)}\right| b^{2}}{2} \\
\dot{R}_{p b}^{(\alpha)}=c_{\text {void } 12} \frac{c_{a n n h 1} \rho_{p b}^{(\alpha)}\left|\dot{\gamma}_{\gamma}^{i n(\alpha)}\right| b^{2}}{2} \\
\dot{R}_{\gamma^{\prime}}^{(\alpha)}=c_{\text {void } 22} \frac{c_{a n n h 2} \rho_{\gamma^{\prime}}^{(\alpha)}\left|\dot{\gamma}_{\gamma^{\prime}}^{\text {in }(\alpha)}\right| b^{2}}{2}
\end{array}\right.
$$

the damage parameter at a time $t$ is calculated by considering the contributions of all the voids that form in the $\gamma$ phase, the $\gamma^{\prime}$ phase and in the $\gamma / \gamma^{\prime}$ interface

$$
D=\left(\int_{t=0}^{t=t}\left(\sum_{\alpha=1}^{12} \dot{R}_{\gamma}^{(\alpha)}+\sum_{\alpha=1}^{12} \dot{R}_{p b}^{(\alpha)}+\sum_{\alpha=13}^{24} \dot{R}_{\gamma^{\prime}}^{(\alpha)}\right) d \tau\right)^{\frac{2}{3}}
$$

the exponent $2 / 3$ converts the volume ratio to the effective crosssectional area of shearing. Finally the effective applied resolved shear stress becomes:

$$
\tau^{(\alpha)}=\frac{\tau_{\text {applied }}^{(\alpha)}}{1-D}
$$

\section{Composition sensitive diffusivity term in flow rule}

In hot section industrial gas turbine components that operate at high temperatures, diffusion of atoms, which is a thermallyinduced phenomenon, is a significant factor in controlling the creep response of the material. Primary creep is a combination of dislocation glide and climb in $\gamma$ channels and at $\gamma / \gamma^{\prime}$ interfaces. Climb of dislocations is a diffusion-controlled and therefore thermally activated mechanism necessary for dislocations to proceed gliding in channels. Additionally, during tertiary creep, once the $a<11 \overline{2}>$ dislocation ribbons are formed by dissociation into Shockley partials, it is likely that shearing of the $\gamma^{\prime}$ precipitates is controlled by diffusion due to the atomic adjustments required at the onset of dislocation movements at $\gamma^{\prime}$ phase [41, 42]. As was previously discussed, rafting of the microstructure also involves interdiffusion of the atoms between the vertical and horizontal channels. Therefore, introducing a diffusivity parameter in the flow rule of $\gamma$ channel provides the means to rigorously describe and capture physics of creep. The diffusivity parameter is defined by equation(11). To determine this parameter and incorporate it in the flow rule, a study on effective diffusivity of the system is conducted by means of kinetic software DICTRA interfaced with thermodynamic software Thermo-Calc [43] which performs all the required thermodynamic calculations.

Estimation of effective diffusivity with DICTRA

DICTRA is a software for simulation of diffusion-controlled processes such as homogenization of alloys, micro-segregation during solidification, and coarsening of precipitates in multicomponent alloys through solving Fick-Onsager law [44, 45] 


$$
\tilde{J}_{i}=-\sum_{j=1}^{n-1} \tilde{D}_{i j} \frac{\partial c_{j}}{\partial z}
$$

where $\tilde{J}_{i}$ is the atomic flux and $\widetilde{D}_{i j}$ is $(n-1)^{2}$ temperature and composition dependent diffusivity matrix.

The simulations are based on assessed thermodynamic and mobility databases that have been developed using CALPHAD method [46]. The accuracy of the simulations strongly depends on the quality of these databases. In present work TCNi5 [47] thermodynamic and MOBNi2 [48] mobility databases have been used to determine the composition-sensitive effective diffusivity.

The equation to describe the effective diffusivity, as is expected, exhibits an Arrhenius form [49],

$$
D_{\text {eff }}=D_{0, e f f} \exp \left(-\frac{Q_{\text {eff }}}{k T}\right)
$$

where $Q_{e f f}$ and $D_{0, e f f}$ are the activation energy and preexponential factor for effective diffusion coefficient, respectively, and can be described by arithmetic and harmonic mean equations such that composition sensitivity is captured and the contribution of each element to the effective values is balanced by their atomic concentration, $C_{m}[50]$,

$$
\begin{aligned}
Q_{\text {eff }} & =\sum_{m} C_{m} Q_{N i-m} \\
D_{0, e f f} & =\frac{1}{\sum_{m} \frac{C_{m}}{D_{0, N i-m}}}
\end{aligned}
$$

in these equations, $Q_{N i-m}$ and $D_{0, N i-m}$ are the activation energy and the pre-exponential factor of element $m$ and $\mathrm{Ni}$ interdiffusion coefficient (chemical diffusivity), respectively.

Diffusivity paths are essentially created in $\gamma$ channels. Moreover, vacancy exchange and dislocation climb during rafting and creep takes place in the disordered $\gamma$ phase. Hence, effective diffusivity of the material is equivalent to the effective diffusivity in $\gamma$ channels.

In order to estimate the composition sensitivity of introduced parameters and evaluate the influence of Re content variation, three compositions with different Re contents ( $0 \% \mathrm{Re}, 3 \% \mathrm{Re}$ and $6 \% \mathrm{Re})$ were defined in addition to $\mathrm{CMSX}-8$ (1.5\% Re). Calculations on composition segregation in $\gamma$ and $\gamma^{\prime}$ phases were carried out at $1000^{\circ} \mathrm{C}$ for each system in DICTRA. Chemical compositions of the $\gamma$ channels for each unique system are presented in

Table II. The results enabled defining of the binary Ni-m couple based on the corresponding composition of element $\mathrm{m}$ in $\gamma$ phase. Interdiffusion coefficients of the binary systems were obtained from the $\mathrm{MOBNi2}$ mobility database in the temperature range of $700^{\circ} \mathrm{C}$ to $1100^{\circ} \mathrm{C}$ using the equilibrium compositions (

Table II). By fitting the chemical diffusivity values to an Arrhenius type relation as a function of temperature, desired diffusion parameters including $Q_{N i-m}$ and $D_{0, N i-m}$ were acquired for all binary systems. The effective diffusivity parameter of Eq. (30) for different Re content compositions were estimated using Eq. (31) and (32). The calculated values are tabulated in Table III.
This method has shown to have good agreement with experimental results. For CMSX-4®, the calculated value of $Q_{e f f}$ is $271.7 \mathrm{~kJ} / \mathrm{mol}$ compared to the experimentally measured value of $272.4 \mathrm{~kJ} / \mathrm{mol}$ [50].

Table II. Equilibrium composition in wt. $\%$ of the $\gamma$ phase at $1000^{\circ} \mathrm{C}$ (balance $\mathrm{Ni}$ )

\begin{tabular}{|c|c|c|c|c|}
\hline & $0 \% \mathrm{Re}$ & $1.5 \% \mathrm{Re}$ & $3 \% \mathrm{Re}$ & $6 \% \mathrm{Re}$ \\
\hline $\mathrm{Cr}$ & 10.0 & 10.0 & 10.3 & 10.7 \\
\hline $\mathrm{Co}$ & 18.6 & 19.0 & 19.4 & 20.0 \\
\hline $\mathrm{Mo}$ & 1.1 & 1.2 & 1.2 & 1.3 \\
\hline $\mathrm{W}$ & 15.0 & 15.4 & 15.8 & 16.6 \\
\hline $\mathrm{Al}$ & 2.8 & 2.5 & 2.2 & 1.7 \\
\hline $\mathrm{Ti}$ & 0.3 & 0.2 & 0.19 & 0.1 \\
\hline $\mathrm{Ta}$ & 1.9 & 1.5 & 1.2 & 0.7 \\
\hline $\mathrm{Re}$ & 0.0 & 3.2 & 606 & 13.7 \\
\hline $\mathrm{Hf}$ & 0.02 & 0.02 & 0.02 & 0.01 \\
\hline
\end{tabular}

Figure 5 illustrates effective diffusivity versus temperature for compositions with distinct Re content following equation (30). It is observed that increase in the refractory element $\mathrm{Re}$ is accompanied by a reduction in the effective diffusivity and temperature sensitivity of the material.
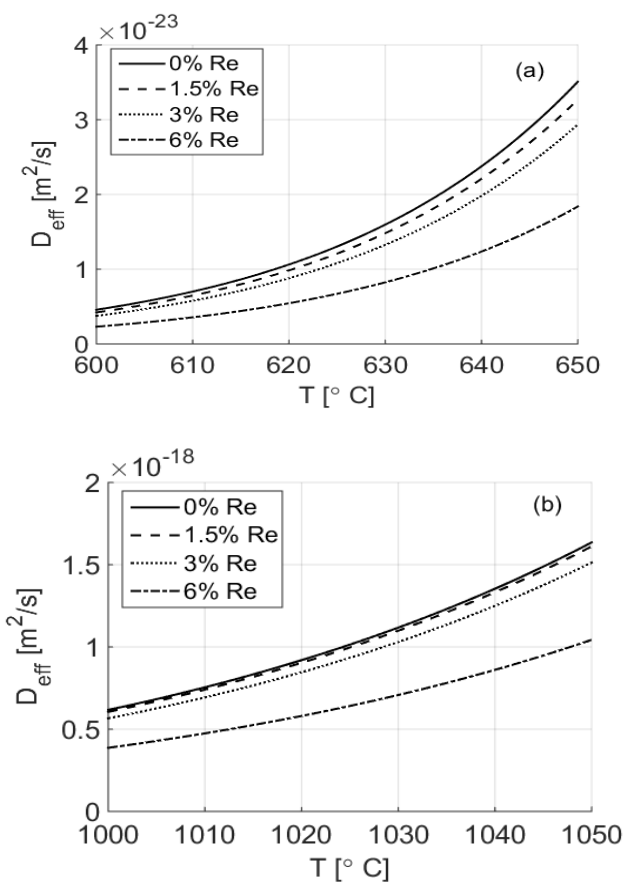

Figure 5. Effective diffusivity versus temperature illustrating the effect of various $\mathrm{Re}$ content in temperature range (a) $600-650^{\circ} \mathrm{C}$ (b) $1000-1050^{\circ} \mathrm{C}$.

It is evident from these plots that sensitivity to composition is not linear such that the influence of Re addition is considerably more effective from $3 \%$ to $6 \%$ than it is from $0 \%$ to $3 \%$. As the temperature increases, this effect becomes more pronounced in such a way that by introducing a small amount of Re to the system, a slight decrement of effective diffusivity is observed whereas with higher Re content, diffusion has been decelerated extensively (Figure 5(b)). 
Table III. Effective diffusivity parameters for four composition combinations.

\begin{tabular}{|c|c|c|c|c|}
\hline & $0 \% \operatorname{Re}$ & $1.5 \% \mathrm{Re}$ & $3 \% \mathrm{Re}$ & $6 \% \mathrm{Re}$ \\
\hline $\mathrm{Q}_{\text {eff }}(\mathrm{kJ} / \mathrm{mol})$ & 272.9 & 274.7 & 275.4 & 277.8 \\
\hline $\mathrm{D}_{0, \text { eff }}\left(\mathrm{m}^{2} / \mathrm{s}\right)$ & $9.69 \mathrm{E}-8$ & $1.09 \mathrm{E}-7$ & $1.13 \mathrm{E}-7$ & $9.73 \mathrm{E}-8$ \\
\hline
\end{tabular}

So far, the effective diffusivity of CMSX-8 has been characterized as a function of composition and temperature. Thereafter, effective terms can be incorporated in the diffusivity parameter of equation (7) resulting in a composition and temperature sensitive flow rule. The constitutive model was calibrated against experimental results with effective activation energy being substituted as $Q_{0}(\xi)$ in equation (11). By calibrating the model to available experimental data, the pre-exponential factor $A(T, \xi)$ was found to be a function of temperature; conceptually it could also be a function of the composition of the alloy using this coefficient.

\section{Model predictions}

The model material parameters were calibrated from creep response data at temperatures ranging from 750 to $1037^{\circ} \mathrm{C}$ and stresses ranging from 124 to $700 \mathrm{MPa}$. This range of test conditions allows for the model be calibrated for both primary and tertiary deformation regimes.
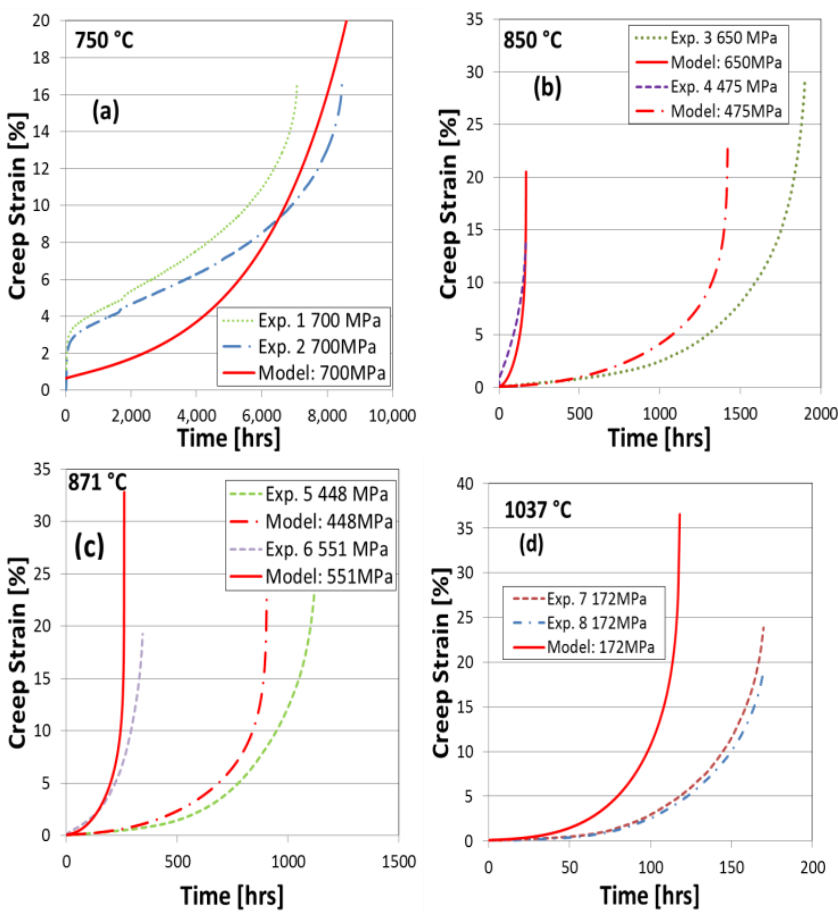

Figure 6. Creep response under for different loading conditions

As shown in Figure 6, the model readily captures all regimes of creep for CMSX-8. In Figure 6 (a) it accurately predicts that the deformation will show significant primary creep; which is in agreement with data from Figure 4. At this higher stress level there is enough force for dislocations to cut through the $\gamma$ ' precipitates thus promoting primary creep. Furthermore note that in Figure 6 (b) and Figure 6 (c) the deformation shows some primary creep but is largely dominated by tertiary creep also consistent with Figure 4. At the highest temperature $1037^{\circ} \mathrm{C}$, there is no visible primary creep and the deformation is completely dominated by tertiary creep as seen in Figure 6 (d). The model parameters constants used in this work are given in Table IV and Table V.

A novel feature of this model is the incorporation of a composition sensitive diffusivity parameter. The form of $A(T, \xi)$ in Eq.(11) is assumed to have a power law,

$$
A(T, \xi)=C_{1}(\xi)\left(\frac{T}{T_{\text {melt }}}\right)^{C_{2}}
$$

where $T$ is given in degrees Kelvin and $\xi$ is used to indicate that the parameter is a function of the composition of the alloy. $C_{l}(\xi)$ and $C_{2}$ were found to be $1.59 \times 10^{8}$ and -23 , respectively.

Table IV. Fundamental microstructure parameters

\begin{tabular}{|c|c|c|c|}
\hline \multicolumn{2}{|c|}{$\begin{array}{c}\text { Fundamental } \\
\text { parameters }\end{array}$} & \multicolumn{2}{c|}{$\begin{array}{c}\text { Microstructure } \\
\text { parameters }\end{array}$} \\
\hline$b$ & $2.49(\AA)$ & \multicolumn{2}{c|}{ Virgin microstructure: } \\
\hline$F_{\text {attack }}$ & $1 \times 10^{12}[1 / \mathrm{s}]$ & $f_{\gamma}$ & 0.3 \\
\hline$\gamma_{A P B}$ & $0.111\left[\mathrm{~J} / \mathrm{m}^{2}\right]$ & $f_{\gamma}$ & 0.7 \\
\hline$\delta$ & -0.002 & $L_{\gamma}$ & $0.14 \times 10^{-6}[\mathrm{~m}]$ \\
\hline$T_{\text {melt }}$ & $1726[\mathrm{~K}]$ & $L_{\gamma}$ & $0.74 \times 10^{-6}[\mathrm{~m}]$ \\
\hline
\end{tabular}

Table V. Parameters fitted to creep deformation data

\begin{tabular}{|r|l|r|l|}
\hline$c_{\text {annhl }}$ & 10.0 & $c_{\text {pass } 11}$ & 0.22 \\
\hline$c_{\text {annh }}^{\text {pbd }}$ & 10.0 & $c_{\text {pass } 22}$ & 1.20 \\
\hline$c_{\text {annh2 } 2}$ & 5.0 & $c_{\text {pass } 21}$ & 0.15 \\
\hline$c_{\text {Fdis }}$ & 10.0 & $c_{\text {vcl }}$ & 1.0 \\
\hline$c^{i c b}$ & 5.0 & $c_{\text {vc } 2}$ & $8.4 \times 10^{-1}$ \\
\hline$c_{\text {iumpl }}$ & $6 \times 10^{-2}$ & $c_{\text {voidl1 }}$ & $8 \times 10^{3}$ \\
\hline$c_{\text {jump } 2}$ & $6 \times 10^{-2}$ & $c_{\text {void } 22}$ & $1 \times 10^{3}$ \\
\hline$c_{\text {misfit }}$ & $180[1 / \mathrm{mol}]$ & $c_{\text {void } 21}$ & $8 \times 10^{3}$ \\
\hline$c_{\text {mult } 1}$ & $3 \times 10^{-3}$ & $Q_{\text {slip }}^{110}$ & $360[\mathrm{~kJ} / \mathrm{mol}]$ \\
\hline$c_{\text {mult }}^{\text {pbd }}$ & $1 \times 10^{-3}$ & $Q_{\text {slip }}^{112}$ & $105[\mathrm{~kJ} / \mathrm{mol}]$ \\
\hline$c_{\text {mult } 2}$ & $9.3 \times 10^{-4}$ & $\left.\rho_{\gamma}^{(\alpha)}\right|_{t=0}$ & $1 \times 10^{11}\left[1 / \mathrm{m}^{2}\right]$ \\
\hline$c_{\text {mult } 21}$ & $5 \times 10^{-16}$ & $\left.\rho_{\gamma}^{(\alpha)}\right|_{t=0}$ & $0\left[1 / \mathrm{m}^{2}\right]$ \\
\hline$c_{\text {oro }}$ & $2.2 \times 10^{-2}$ & $\rho_{\gamma}^{r e f}$ & $1.0 \times 10^{13}$ \\
\hline & & & \\
\hline
\end{tabular}

Rafting of the microstructure was not considered in this model. In the aging studies, rafting became observable for temperatures of $950{ }^{\circ} \mathrm{C}$ and above. Rafting plays a role in the response of creep and more research is needed to incorporate it in the model. Modeling of the evolution of the microstructure during rafting is necessary to completely account for the deformation of CMSX-8. This could be accomplished by utilizing equation (3) as an evolution equation for the morphology of the microstructure and then modifying the model to consider a parameter that instead of assuming a cuboidal microstructure, where the hardening can be described exclusively by the length of the precipitates, it utilizes a more elaborate formulation where the aspect ratio the precipitates in the evolved microstructure is considered instead, as done in reference [5].

\section{Conclusions and future work}

We presented three different tools that can enable the ICME of Ni-base superalloys. In the first tool equations that can predict the microstructure evolution during aging were developed, these equations can be used to determine the geometry of the 
microstructure which can serve as an input in CVP models. In the second tool a composition sensitive CVP model is developed that can consider the $\mathrm{Re}$ content in the alloy and is capable of predicting the creep behavior of CMSX-8. In the final tool, computational kinetics modeling is used to determine the value of composition-sensitive parameters in the CVP model. Although still in its infancy, the integration and further development of the tools presented can help pave the way for alloy design and design of components with controlled spatial microstructure variations. In future work the aging equations will be used in the CVP to explicitly model the rafting regime in this alloy.

\section{Acknowledgments}

This work is supported by U.S. Department of Energy, National Energy Technology Laboratory, University Turbine Systems Research (UTSR) Program, Grant DE-FE0011722, and Siemens Energy Inc., Orlando, FL.

Disclaimer: "This report was prepared as an account of work sponsored by an agency of the United States Government. Neither the United States Government nor any agency thereof, nor any of their employees, makes any warranty, express or implied, or assumes any legal liability or responsibility for the accuracy, completeness, or usefulness of any information, apparatus, product, or process disclosed, or represents that its use would not infringe privately owned rights. Reference herein to any specific commercial product, process, or service by trade name, trademark, manufacturer, or otherwise does not necessarily constitute or imply its endorsement, recommendation, or favoring by the United States Government or any agency thereof. The views and opinions of authors expressed herein do not necessarily state or reflect those of the United States Government or any agency thereof."

\section{References}

1. Wahl, J. B. and K. Harris. New Single Crystal Superalloys, CMSX-7 and CMSX-8. in Superalloys 2012 Seven Springs Mountain Resort,Seven Springs, PA: TMS.

2. Harris, K., et al., Development of the rhenium containing superalloys CMSX-4 and CM 186 LC for single crystal blade and directionally solidified vane applications in advanced turbine engines, in Superalloys 1992, R. W. Stusrud S. D. Antolovich, R. A. MacKay, D. L. Anton, T. Khan, R. D. Kissinger, D. L. Klarstrom, Editor. 1992, The Minerals, Metals and Materials Society (TMS): Warrendale, PA. p. 297-306.

3. Wahl, J. B. and K. Harris, NEW SINGLE CRYSTAL SUPERALLOYS, CMSX (R)-7 AND CMSX (R)-8. Superalloys 2012, 2012: p. 179-188.

4. Fullwood, D. T., S. R. Niezgoda and S. R. Kalidindi, Microstructure reconstructions from 2-point statistics using phase-recovery algorithms. Acta Materialia, 2008. 56(5): p. $942-$ 948.

5. Kirka, Michael, Thermomechanical behavior of a directionally solidified nickel-base superalloy in the aged state, in The George W. Woodruff School of Mechanical Engineering. 2014, Georgia Institute of Technology: Atlanta, Georgia p. 230.

6. Jaroszewicz, J., et al., Characterization of Single-Crystal Dendrite Structure and Porosity in Nickel-Based Superalloys
Using X-Ray Micro-Computed Tomography. Euro Superalloys 2010, 2011. 278: p. 66-71.

7. Epishin, A., et al., Effects of segregation in nickel-base superalloys: Dendritic stresses. Superalloys 2004, 2004: p. 537543.

8. Reed, Roger C., Superalloys - Fundamentals and Applications. 2006: Cambridge University Press.

9. Epishin, A., et al., Microstructural degradation of CMSX-4: Kinetics and effect on mechanical properties. Superalloys 2008, 2008: p. 725-731.

10. Reed, R. C., The superalloys fundamentals and applications. 1st ed. 2006: Cambridge University press.

11. Fullwood, D. T., et al., Microstructure sensitive design for performance optimization. Progress in Materials Science, 2010. 55(6): p. 477-562.

12. Nabarro, F. R. N., Rafting in superalloys. Metallurgical and Materials Transactions a-Physical Metallurgy and Materials Science, 1996. 27(3): p. 513-530.

13. Tinga, T., W. A. M. Brekelmans and M. G. D. Geers, Directional coarsening in nickel-base superalloys and its effect on the mechanical properties. Computational Materials Science, 2009. 47(2): p. 471-481.

14. Matan, N., et al., On the kinetics of rafting in CMSX-4 superalloy single crystals. Acta Materialia, 1999. 47(7): p. 20312045.

15. Mushongera, L. T., et al., Effect of Re on directional gamma 'coarsening in commercial single crystal Ni-base superalloys: A phase field study. Acta Materialia, 2015. 93: p. 60-72.

16. Asaro, R. J., Crystal Plasticity. Journal of Applied MechanicsTransactions of the Asme, 1983. 50(4B): p. 921-934.

17. Tinga, Tiedo, W. A. Brekelmans and Marc G. D. Geers, Cube slip and non-Schmid effects in single crystal Ni-base superalloys. Modeling and simulation in material science and engineering 2010. 18.

18. Staroselsky, A. and B. N. Cassenti, Creep, plasticity, and fatigue of single crystal superalloy. International Journal of Solids and Structures. 48: p. 2060-2075.

19. Ma, A., D. Dye and R. C. Reed, A model for the creep deformation behaviour of single-crystal superalloy CMSX-4. Acta Materialia, 2008. 56(8): p. 1657-1670.

20. Pollock, T. M. and A. S. Argon, Creep Resistance of CMSX-3 Nickel-Base Superalloy Single-Crystals. Acta Metallurgica Et Materialia, 1992. 40(1): p. 1-30.

21. Pollock, T. M. and A. S. Argon, Creep resistance of CMSX-3 nickel base superalloy single crystals. Acta Metallurgica et Materialia, 1992. 40(1): p. 1-30. 
22. Rae, C., et al., Dislocations in a Ni-based superalloy during low temperature creep. Eurosuperalloys 2014 - 2nd European Symposium on Superalloys and Their Applications, 2014. 14.

23. Zhang, J. X., et al., Creep deformation mechanisms in some modern single-crystal superalloys. Superalloys 2004, 2004: p. 189-195.

24. Koizumi, Yutaka, et al., Development of next-generation $\mathrm{Ni}$ base single cyrstal superalloys. Superalloys 2004, 2004: p. 35-43.

25. Ma, A. and F. Roters, A constitutive model for fcc single crystals based on dislocation densities and its application to uniaxial compression of aluminium single crystals. Acta Materialia, 2004. 52(12): p. 3603-3612.

26. Busso, E. P. and F. A. McClintock, A dislocation mechanicsbased crystallographic model of a B2-type intermetallic alloy. International Journal of Plasticity, 1996. 12(1): p. 1-28.

27. Österle, W., et al., Modelling the orientation and direction dependence of the critical resolved shear stress of nickel-base superalloy single crystals. Acta Materialia, 2000. 48(3): p. 689700.

28. Sheh, M. Y. and D. C. Stouffer, A Crystallographic Model for the Tensile and Fatigue Response for Rene $\mathrm{N} 4$ at $982^{\circ} \mathrm{C}$. Journal of Applied Mechanics, 1990. 57(1): p. 25-31.

29. Meric, L., P. Poubanne and G. Cailletaud, Single Crystal Modeling for Structural Calculations: Part 1 - Model Presentation. Journal of Engineering Materials and Technology, 1991. 113: p. 162-170.

30. Meric, L. and G. Cailletaud, Single Crystal Modeling for Structural Calculations: Part 2 - Finite Element Implementation. Journal of Engineering Materials and Technology, 1991. 113: p. 171-182.

31. Shenoy, M. M., et al., Thermomechanical fatigue behavior of a directionally solidified Ni-base superalloy. Journal of Engineering Materials and Technology-Transactions of the Asme, 2005. 127(3): p. 325-336.

32. Song, J. E. and D. L. McDowell, Grain Scale Crystal Plasticity Model with Slip and Microtwinning for a Third Generation Ni-Base Disk Alloy. Superalloys 2012, 2012: p. 159166.

33. Shenoy, Mahesh, Yustianto Tjiptowidjojo and David McDowell, Microstructure-sensitive modeling of polycrystalline IN 100. International Journal of Plasticity, 2008. 24(10): p. 16941730.

34. Wang, A. J., et al., Microstructure-based multiscale constitutive modeling of gamma-gamma' nickel-base superalloys. International Journal for Multiscale Computational Engineering, 2006. 4(5-6): p. 663-692.

35. Zhao, L. G., N. P. O'Dowd and E. P. Busso, A coupled kinetic-constitutive approach to the study of high temperature crack initiation in single crystal nickel-base superalloys. Journal of the Mechanics and Physics of Solids, 2006. 54(2): p. 288-309.
36. Dumoulin, S., et al., A multiscale approach for coupled phenomena in fcc materials at high temperatures. Philosophical Magazine, 2003. 83(31-34): p. 3895-3916.

37. Fedelich, B., et al., Constitutive modelling of creep degradation due to rafting in single-crystalline Ni-base superalloys. Materials Science and Engineering a-Structural Materials Properties Microstructure and Processing, 2009. 510-11: p. 273-277.

38. Fedelich, B., et al., Rafting during High Temperature Deformation in a Single Crystal Superalloy: Experiments and Modeling. Superalloys 2012, 2012: p. 491-500.

39. Basoalto, H., et al., A generic microstructure-explicit model of creep in nickel-base superalloys. Superalloys 2004, 2004: p. 897906.

40. Kocks, U. F. and H. Mecking, Physics and phenomenology of strain hardening: the FCC case. Progress in Materials Science, 2003. 48(3): p. 171-273.

41. Rae, C. M. F. and R. C. Reed, Primary creep in single crystal superalloys: Origins, mechanisms and effects. Acta Materialia, 2007. 55(3): p. 1067-1081.

42. Yang, H., M. S. Huang and Z. H. Li, The influence of vacancies diffusion-induced dislocation climb on the creep and plasticity behaviors of nickel-based single crystal superalloy. Computational Materials Science, 2015. 99: p. 348-360.

43. Andersson, J. O., et al., THERMO-CALC \& DICTRA, computational tools for materials science. Calphad-Computer Coupling of Phase Diagrams and Thermochemistry, 2002. 26(2): p. 273-312.

44. Borgenstam, A., et al., DICTRA, a tool for simulation of diffusional transformations in alloys. Journal of Phase Equilibria, 2000. 21(3): p. 269-280.

45. Wang, C. P., et al., Diffusion mobilities in the $f c c A g-C u$ and Ag-Pd alloys. Calphad-Computer Coupling of Phase Diagrams and Thermochemistry, 2012. 37: p. 57-64.

46. Agren, J., Calculation of phase diagrams: Calphad. Current Opinion in Solid State \& Materials Science, 1996. 1(3): p. 355360.

47. McDowell, David L., Viscoplasticity of heterogeneous metallic materials. Materials Science and Engineering: R: Reports, 2008. 62(3): p. 67-123.

48. Giordana, M. F., et al., Micromechanical modeling of the cyclic softening of EUROFER 97 steel. Procedia Engineering, 2011. 10: p. 1268-1273.

49. Zhu, Z., et al., A model for the creep deformation behaviour of nickel-based single crystal superalloys. Acta Materialia, 2012. 60(12): p. 4888-4900.

50. Ai, C., et al., Application of a modified Ostwald ripening theory in coarsening of gamma 'phases in Ni based single crystal superalloys. J. of Alloys and Compounds, 2015. 632: p. 558-562. 